\title{
CHARM PRODUCTION AT LARGE RAPIDITIES IN P+P AND D+AU COLLISIONS AT PHENIX AT RHIC
}

\author{
XIAORONG WANG FOR PHENIX COLLABORATION \\ Physics Department, New Mexico State University, Las Cruces, NM88003, USA \\ Hua-Zhong Normal University, Wuhan 430079, P.R. China \\ E-mail: xrwang@bnl.gov
}

\begin{abstract}
We study charm production through dimuon and single muon measurements at forward and backward rapidities in $\mathrm{p}+\mathrm{p}$ and $\mathrm{d}+\mathrm{Au}$ collisions with the PHENIX muon detectors. We also compare open charm to $J / \psi$ yields in the forward and backward rapidities in $\mathrm{d}+\mathrm{Au}$ collisions and study the origin of the large forward and backward asymmetry in open charm production observed by the PHENIX experiment.
\end{abstract}

\section{Introduction:}

Charm quarks are believed to be mostly created from initial gluon fusion in hadronic collisions. Since they are massive, heavy flavor hadrons are proposed to be ideal probes to study the early stage dynamics in heavy-ion collisions. Measurements of heavy quark production in $\mathrm{p}+\mathrm{p}$ collisions serve as important tests for perturbative Quantum Chromo Dynamics (pQCD).

$J / \psi$ and open charm productions are two of the most important hard probes of the hot dense matter created in $\mathrm{Au}+\mathrm{Au}$ collisions. It is necessary to understand their production in the cold nuclear medium in $\mathrm{d}+\mathrm{Au}$ collisions as a reference in their production in $\mathrm{Au}+\mathrm{Au}$ collisions.

Since the initial formation of both open and closed charm is sensitive to initial gluon densities, then gluon structure functions, shadowing or antishadowing and initial state energy loss will all effect their production. During the hadronization, the $J / \psi$ production mechanism is different from that of open charm. In the final state, the $J / \psi$ can be disassociated or absorbed, but for open charm, the main nuclear medium effect is final state multiple scattering and energy loss. The comparison of open charm and the $J / \psi$ production will help us to understand $J / \psi$ production mechanism and disentangle different initial state and final state nuclear medium effects.

In $\sqrt{s_{N N}}=200 \mathrm{GeV} \mathrm{d}+\mathrm{Au}$ collisions at RHIC, measurements at for- 
ward rapidity (deuteron direction) probe the shadowing region while the anti-shadowing region is probed in backward rapidity. Recent models of gluon shadowing ${ }^{1}$, color glass condensate ${ }^{2}$ and recombination ${ }^{3}$ are implemented to understand open charm production at forward rapidity.

\section{Charm measurement at PHENIX}

The PHENIX experiment ${ }^{4}$ has measured $J / \psi$ and open charm production through observation of dilepton and semi-leptonic decays at forward and backward rapidity with the PHENIX muon spectrometers, covering both forward and backward directions in the rapidity range of $1.2<|\eta|<2.4$.

\section{Open Charm results in $p+p$ and $d A u$ collisions}

A PYTHIA simulation shows around $75 \%$ of prompt muons with $p_{T}>$ $0.9 \mathrm{GeV} / c$ come from open charm decay in $\mathrm{d}+\mathrm{Au}$ collisions, while $11 \%$ come from open bottom decay. Prompt muons are produced close to the collision vertex. We can separate heavy flavor decays and light hadron decays experimentally by studying the shape of the vertex distribution.
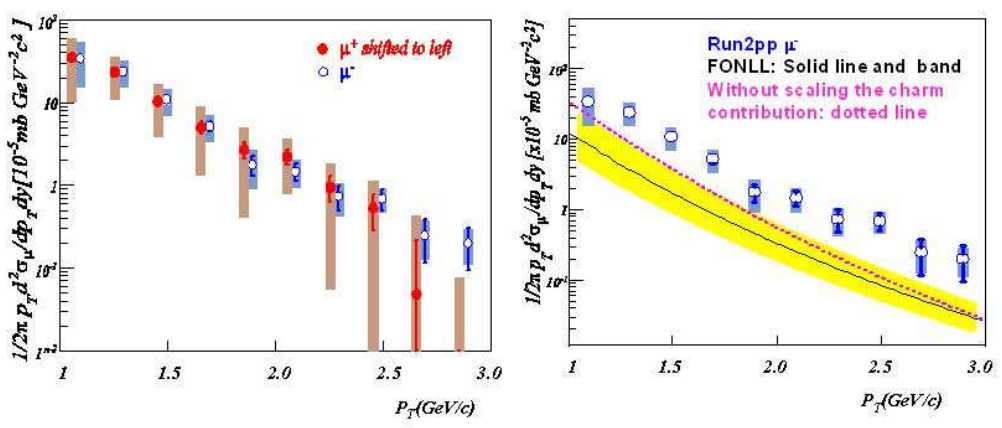

Figure 1. Left: $p_{T}$ spectrum of prompt muons. Error bars indicate statistical errors and shaded bands indicate systematic errors. Right: The measured $p_{T}$ spectrum of negative prompt muons, the PYTHIA prediction without scaling the charm contribution (dotted line), and a FONLL calculation (solid line with systematic error band).

The invariant differential cross section for muon candidate production at forward rapidity $(1.5<\eta<1.8)$ has been measured by PHENIX over the transverse momentum range $1<p_{T}<3 \mathrm{GeV} / c$ in $200 \mathrm{GeV}$ p + p collisions ${ }^{5}$. The resulting muon spectrum from heavy flavor decays is compared to 
PYTHIA and a next-to-leading order perturbative QCD calculation, shown in Figure 1. PHENIX muon arm data (at forward and backward rapidity) is compatible with the PHENIX charm measurement at $\mathrm{y}=0{ }^{6}$, and it exceeds predictions from PYTHIA and FONLL.

The nuclear modification factor of $\mathrm{d}+\mathrm{Au}$ collisions is defined as the particle yield per nucleon-nucleon collision relative to the yield in $\mathrm{p}+\mathrm{p}$ collisions. The nuclear modification factors for prompt muons are shown in Figure 2. Prompt muon production shows suppression at forward rapidity and enhancement in the backward direction.
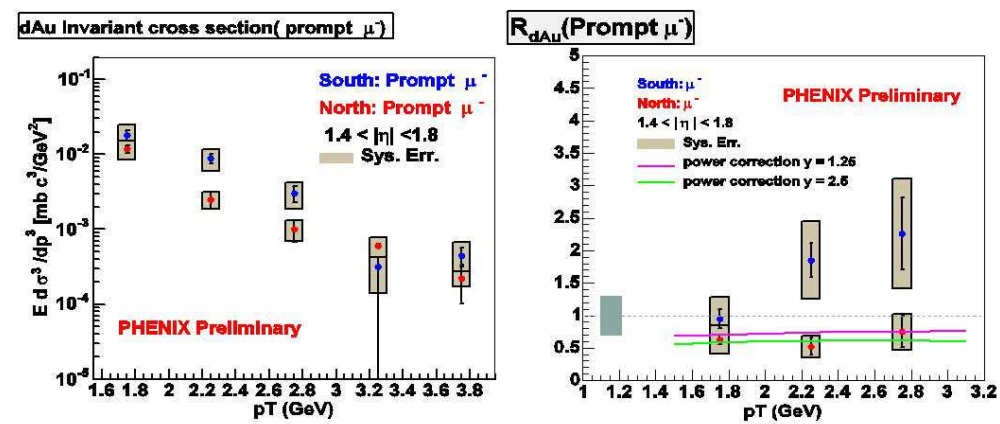

Figure 2. Invariant spectra of the prompt muon (left) and nuclear modification factor of prompt muons(right) in $\mathrm{d}+\mathrm{Au}$ collisions. The theoretical curves are come from power correction model at $\eta=1.25$ and 2.5 .

\section{4. $J / \psi$ results in $\mathrm{p}+\mathrm{p}$ and $\mathrm{dAu}$ collisions}

PHENIX measured $J / \psi$ production in forward, backward and central rapidity in $\mathrm{p}+\mathrm{p}$ and $\mathrm{d}+\mathrm{Au}$ collisions at $200 \mathrm{GeV}^{8}$.

Figure 3a shows the measured pp differential cross section times branching ratio vs rapidity. A fit to a shape generated with PYTHIA is performed, and using a di-lepton branching ratio of $5.9 \%$ gives a total cross section $\sigma_{p p}^{J / \psi}=2.61 \pm 0.20($ fit $) \pm 0.26(a b s) \mu \mathrm{b}$. Figure 3b shows the nuclear modification factor vs rapidity. It is significantly lower at the forward rapidity. Theoretical calculations that include the effects of absorption and shadowing are shown in the Figure 3b. The data favor a modest shadowing rather than the stronger gluon shadowing. 


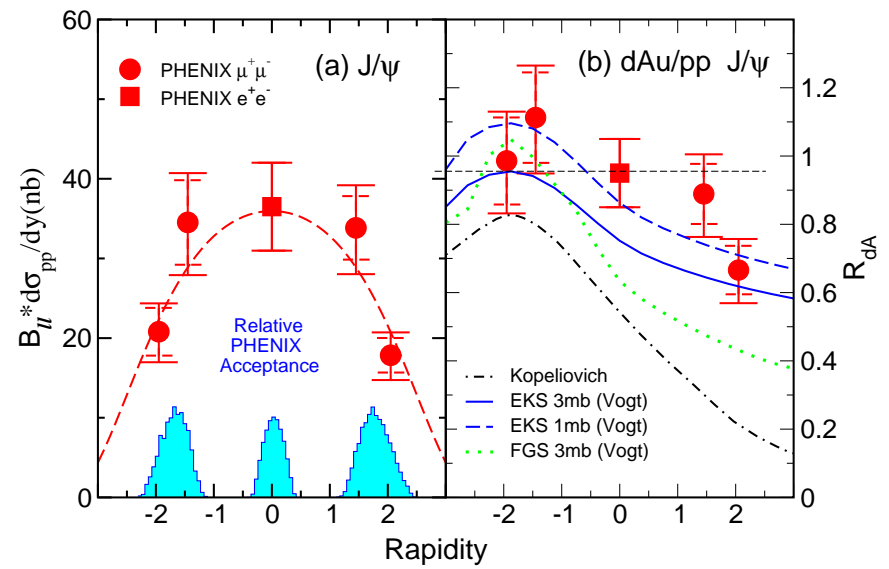

Figure 3. (a) $J / \psi$ differential cross section times di-lepton branching ratio vs rapidity. (b) The minibias $R_{d A u}$ vs rapidity.

\section{Summary and outlook}

We observe a significant cold nuclear medium effect in charm production in forward and backward rapidity in $\mathrm{d}+\mathrm{Au}$ collisions at $200 \mathrm{GeV} / c$. Both open charm and $J / \psi$ results show a suppression in forward rapidity. The open charm data are consistent with CGC and power correction model ${ }^{7}$; the $J / \psi$ data favor shadowing with weak absorption. At backward rapidity, open charm results show enhancement while $J / \psi$ results are consistent with unity.

We need a more precise $\mathrm{d}+\mathrm{Au}$ measurements and more theoretical work to understand the cold nuclear medium effects as a baseline for understanding the hot dense matter produced in $\mathrm{Au}+\mathrm{Au}$ collisions.

\section{References}

1. Eskola, Kolhinen, Vogt, Nucl. Phys. A696 (2001) 729-746.

2. L. McLerran and R Venugopalan, Phys. Rev. D49, 2233(1994); Phys. Rev. D49 3352(1994)

3. R.C. Hwa, C.B.Yang and R.J. Fries, Phys.Rec C71, 024902(2005)

4. K. Adcox et al., Nucl. Instrum. Methods A499, 469(2003)

5. Y. Kwon for PHENIX collaborations, nucl-ex/0510011.

6. S.S. Adler et. al, PHENIX colloborations, Phys. Rev. Lett. 96, 032001 (2006)

7. J. Qiu, I. Vitev, Phys.Lett. B632, (2006)507-511

8. S.S. Adler et. al, PHENIX collaborations, Phys. Rev. Lett 96, 012304 (2006) 